\title{
An Experimental Model of a Acousto-Electromagnetic Sensor for Detecting Land Mines
}

\author{
Waymond R. Scott, Jr.* \\ School of Electrical and Computer Engineering \\ Georgia Institute of Technology \\ Atlanta, GA 30332-0250
}

\author{
James S. Martin \\ School of Mechanical Engineering \\ Georgia Institute of Technology \\ Atlanta, GA 30332-0405
}

\section{Introduction}

A hybrid technique is presented in this paper that simultaneously uses both electromagnetic and acoustic waves in a synergistic manner to detect buried land mines. The configuration of the system currently being studied is shown in figure 1 . The system consists of an electromagnetic radar and an acoustic source. An acoustic surface (Rayleigh) wave is induced in the earth by means of a stationary transducer on the surface. The transducer is placed behind the region being scanned for mines and directs the surface acoustic wave into the region being searched. The surface acoustic wave interacts with the mine and causes both the mine and the surface of the earth to be displaced. The displacement of the mine is different than the earth, because the acoustic properties of the mine are quite different than those of the earth. The displacement of the surface of the earth when a mine is present is different than when it is not present because of the waves scattered from the mine. The electromagnetic radar is used to detect these displacements and, thus, the mine. This idea has been discussed previously $[1,2]$, but it has not been seriously investigated.

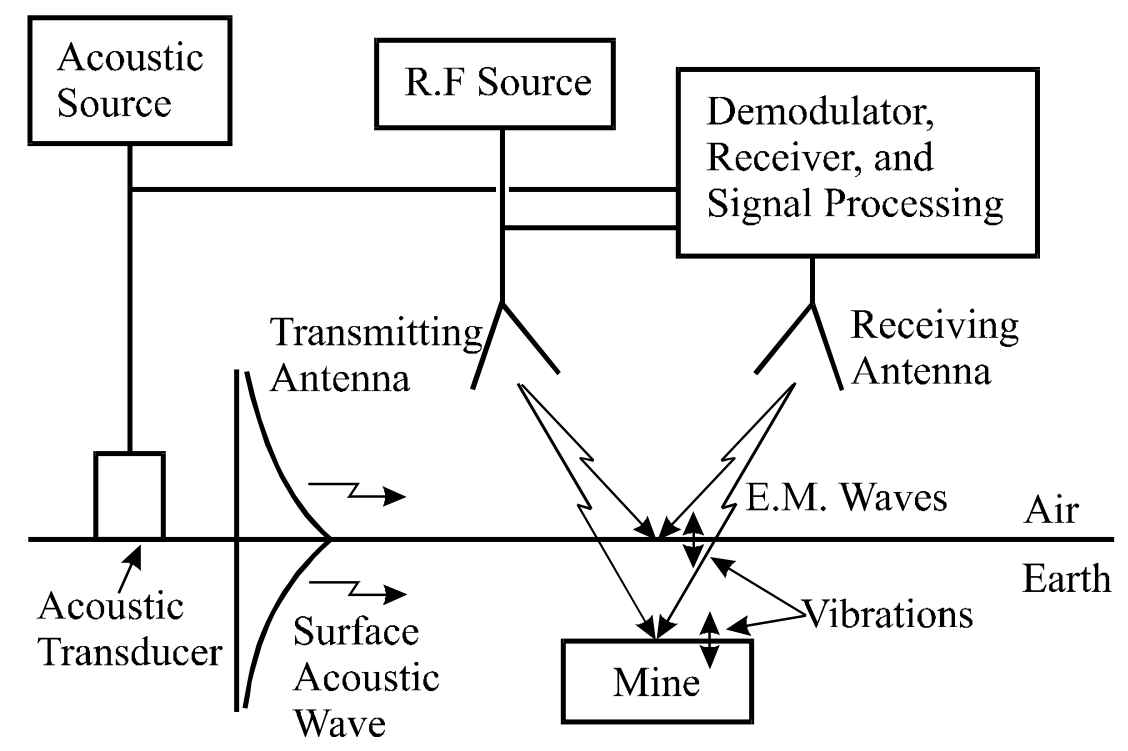

Figure 1. Acousto-electromagnetic mine detection system.

This work is supported in part by the US Army Research Office under contract DAAH04-96-1-0048. 


\section{Experimental Model}

A radar has been designed and built to measure the displacements of the surface of the earth and the mine. The radar radiates electromagnetic waves that are reflected off of the surface of the earth and the mine. The reflected waves are received by the radar, and a homodyne system is used to demodulate the signals. The displacements are determined from these demodulated signals. The two biggest challenges to make this radar perform adequately for the mine detection system are 1) to make it sufficiently sensitive to be able to detect the small vibrations, and 2) to make the spot size (the area on the surface illuminated by the electromagnetic waves) sufficiently small. The radar can measure vibrations as small as $1 \mathrm{~nm}\left(10^{-9} \mathrm{~m}\right)$ as currently configured. To obtain this sensitivity, the radar was designed to minimize the effects of noise, such as the phase noise of the source and the electromagnetic interference from low-frequency magnetic fields. The spot size of the radar must be smaller than approximately one half of a wavelength of the acoustic waves. Currently, a small spot size is obtained by using an open-ended waveguide as the antenna for the radar. This antenna produces a sufficiently small spot size when the open end of the antenna is placed within a few centimeters of the surface. Antennas are being investigated that produce a sufficiently small spot size when they are placed farther from the surface. The radar can be configured in either a monostatic mode or in a bistatic mode as in figure 1. Currently the radar is being used in the monostatic mode to get a smaller spot size, but the radar has been used and has performed well in the bistatic mode. The radar can be operated at frequencies between $2 \mathrm{GHz}$ and $8 \mathrm{GHz}$; however, all of the results presented in this work are made with the radar operating at 8 GHz. The displacements of both the surface of the sand and the mine have been measured with both the radar and an accelerometer. The measurements with the radar are good agreement with those from the accelerometer.

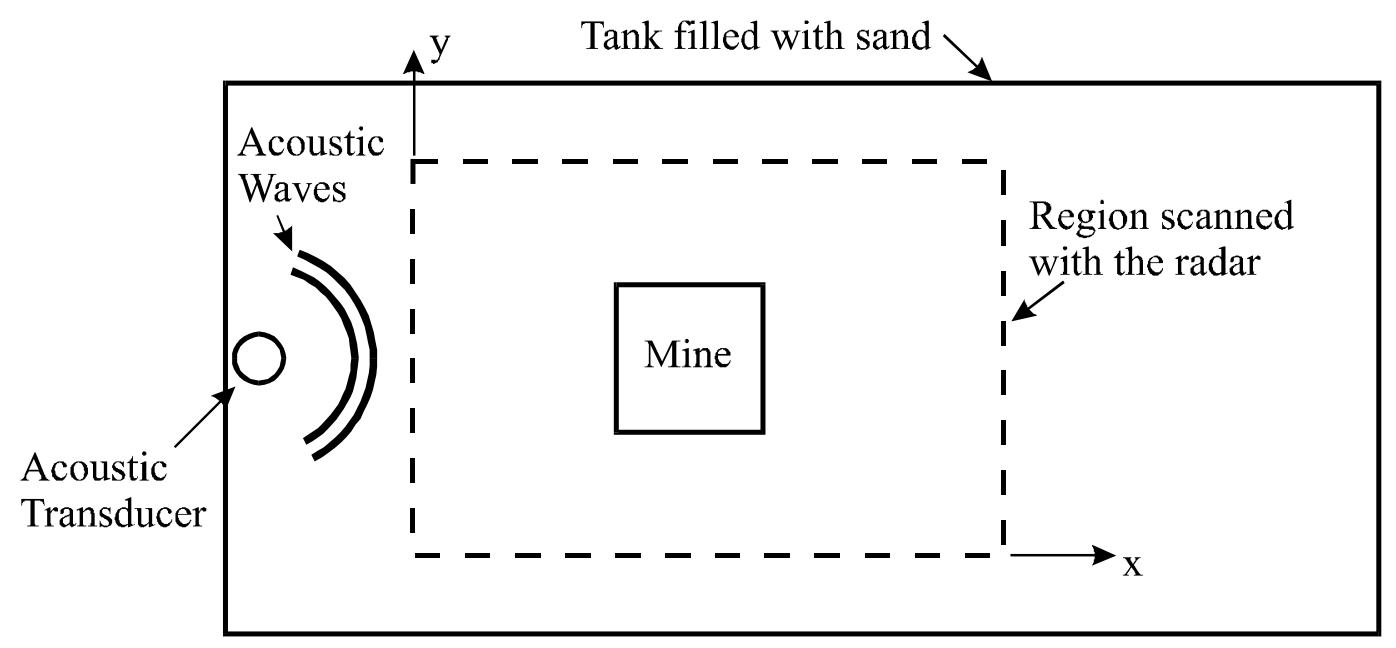

Figure 2 Top view of experimental model.

A drawing of the top view of the experimental model is shown in figure 2. The model consists of a tank that is filled with damp sand that has been packed to a relatively uniform density. The tank is approximately $120 \mathrm{~cm}$ wide, $120 \mathrm{~cm}$ deep, and $240 \mathrm{~cm}$ 
long. A transducer is placed on the surface of the sand and is used to launch the acoustic waves into the sand. The acoustic transducer is excited with a differentiated gaussian pulse with a center frequency of $400 \mathrm{~Hz}$. The transducer has been coupled to the surface so that it preferentially launches surface acoustic waves. These surface waves travel across the surface of the tank and interact with a simulated mine that is buried in the sand. The radar is used to measure the vibrations caused by the acoustic waves. An automated positioner is used to scan the radar over the surface of the sand. The region scanned ( 80 $\mathrm{cm}$ wide by $120 \mathrm{~cm}$ long) with the radar is also indicated on figure 2 . The displacements of the surface of the sand and the mine are measured in this region as a function of time and position. The measurements are made on a uniform rectangular grid of discrete positions in the scanned region. The displacements are measured and recorded as a function of time at each of these points.

\section{Results}

Pseudo color graphs of the amplitude of the displacement of the surface are presented in figures 3 for four times. These results are for a simulated antitank mine buried in the sand. The simulated anti-tank mine made out of Acrylic plastic and is $30 \mathrm{~cm}$ wide, $30 \mathrm{~cm}$ long, and $7.5 \mathrm{~cm}$ height. The top of the mine is flush with the surface of the sand. The position of the mine is indicated by the dotted white line. In these graphs, the color scale goes from black to blue to green to yellow to red to white. The smallest displacements are in black and the largest displacements are in white. At time \#1, the acoustic wave is seen traveling toward the mine. Note that the shape of the wave is spread out and more complex than the differentiated gaussian pulse that is the excitation for the transducer. This is partially due to the manner in which the wave is injected into the sand. Methods for injecting a more time-limited pulse are being investigated. At times \#2 and \#3, the wave has reached the mine, and a portion of the wave is going through the mine while the rest is going around the mine. Notice that the portion of the wave that is going through the mine is ahead of the portion that is going around the mine. This is because the mine is much stiffer than is the sand; thus, the wave appears to travel faster through the mine. The mine actually moves as if were a rigid body. The motion of the mine is essentially a rocking motion excited by the wave motion in the sand. At time \#4, the wave is seen to be still going around the mine; however, a significant portion has passed through the mine. Notice that the wave that went through the mine is still ahead of the wave that went around then mine, and notice that the wave that went through the mine is smaller in amplitude than the one that went around the mine. Also notice that the displacements are smaller above the mine. The waves that are reflected from the mine can also be seen. The location and the shape of the mine are clearly evident in these graphs. Measurements have also been made with the anti-tank mine buried deeper and with simulated antipersonnel mines. The effects of the mine can also be seen in these measurements.

\section{References}

[1] G.S. Smith, "Summary Report: Workshop on New Directions for Electromagnetic Detection of Non-Metallic Mines," Report for U.S. Army BRDEC and ARO, June 1992

[2] C. Stewart, Summary of Mine Detection Research, Vol. I, pp. 172-179, Tech. Report 1636TR, May 1960, U.S. Army Engineering Res. and Devel. Labs, Corps. of Eng., Belvoir, VA. 


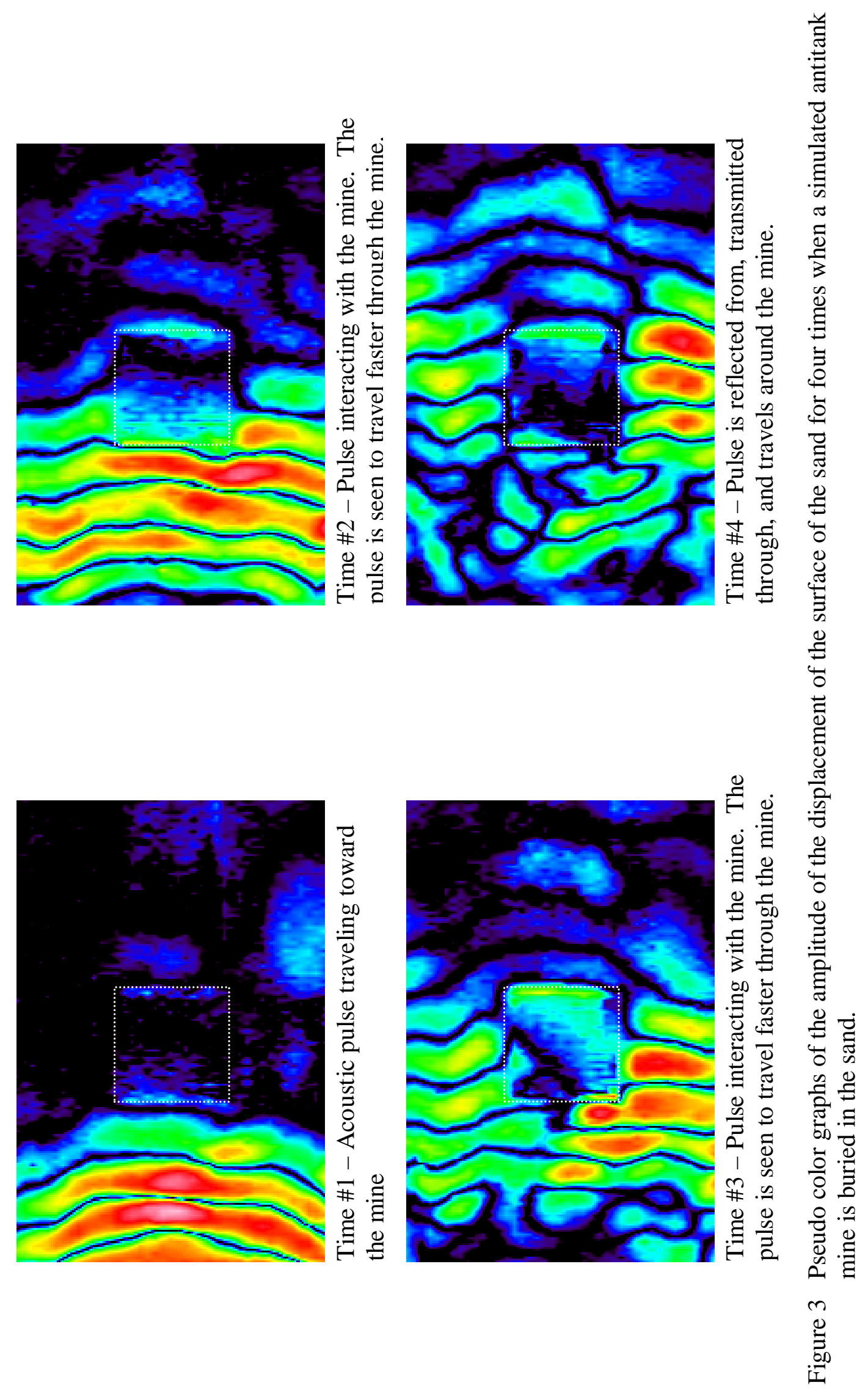

